\title{
FERTILIZACIÓN CON SILICATO DE POTASIO, PARA LA PRODUCCIÓN DE NOCHEBUENA
}

\author{
Potassium silicate fertilization to promote growth of poinsettia plants
}

Alejandro Velasco-Ramírez ${ }^{1 *}$, María Luisa García-Sahagún ${ }^{1}$, Ana Paulina VelascoRamirez ${ }^{1}$, José Pablo Torres-Morán ${ }^{2}$, Alicia de Luna Vega ${ }^{1}$, Salvador González-Luna ${ }^{1}$.

${ }^{1}$ Departamento de Producción Agrícola, ${ }^{2}$ Departamento de Desarrollo Rural Sustentable. Centro Universitario de Ciencias Biológicas y Agropecuarias, Universidad de Guadalajara, Zapopan, Jalisco, CP 45110, México.

*Autor para correspondencia: alejandro.velasco@academicos.udg.mx

\section{Resumen}

Se probaron tres tratamientos de fertilización en el cultivo de nochebuena para medir su efecto en el crecimiento de la planta. Los tratamientos fueron aplicados en drench y en forma foliar. Adicionalmente a los tratamientos de fertilización, se probó un producto a base de Silicato de potasio (Sillisec K $®$ ). Se tomaron datos de tamaño de planta $y$ producción de brotes y flores. Se probaron las diferencias entre los tratamientos, usando la prueba de Kruskall-Wallis y chi cuadrada. Los resultados obtenidos permitieron demostrar la superioridad de todos los tratamientos en comparación con el testigo, siendo el tratamiento a base de fertilización en drench + Sillisec $\mathrm{K}+$ foliar el que produjo mejores resultados.

Palabras clave: Producción de ornamentales, fertilización integral, Euphorbia pulcherrima.

\section{Abstract}

Three fertilization treatments were tested on poinsettia plants in order to measure their effects on plant growth. The treatments were applied by drenching and foliar. In addition to the fertilization treatments, a Potassium Silicate (Sillisec $\mathrm{K}(\circledR)$-based product was also put to the test. Plant sizes, and bud and flower production were measured. Differences among treatments were tested using the Kruskall-Wallis and chi-square test. The results demonstrated the superiority of all three treatments in comparison with the control sample, with the fertilization in drench + Sillisec $K+$ foliar treatment yielding the best results.

Keywords: Ornamental plant production, integral fertilization, Euphorbia pulcherrima.

\section{Introducción}

En México, la producción de nochebuena, se ha convertido en un negocio sumamente rentable y competido. Sin embargo, el manejo de las plantas para su producción y comercialización, particularmente en lo que respecta a la fertilización, no se realiza de una manera selectiva de acuerdo a las condiciones específicas de cada lugar de producción, sino que se adoptan programas de 
fertilización de acuerdo a recomendaciones de las casas comerciales que distribuyen los esquejes enraizados y las variedades que actualmente se utilizan para la producción de esta especie (Figura $1)$.

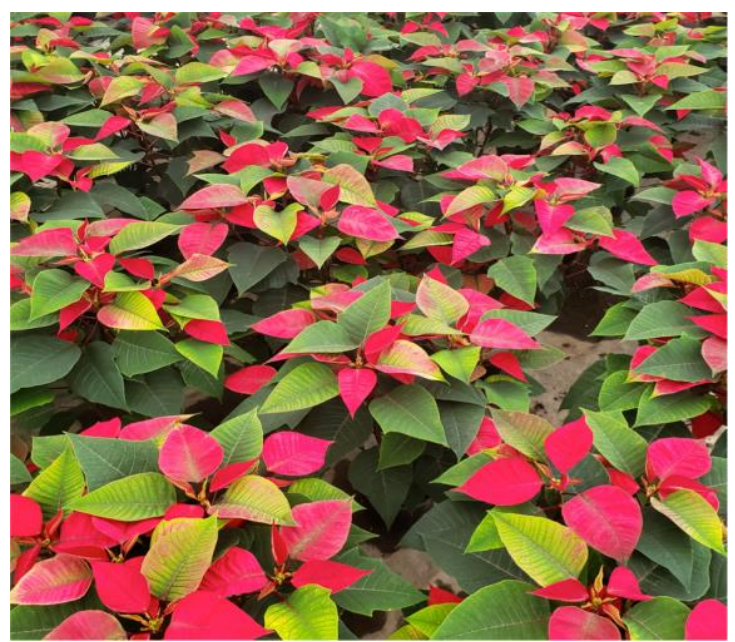

Figura 1. Aspecto general de la coloración de las brácteas en nochebuena

Uno de los métodos más comúnmente usados, se basa en la aplicación de fertilizante líquido a una dosis constante en cada riego, lo cual se ha hecho desde la década de los 90's (Schuch et al., 1995) y se le consideraba un método cómodo y económico. Sin embargo, a medida que crece la contaminación en suelos y agua, se ha considerado importante observar la acumulación de residuos fertilizantes lixiviados y que se acumulan en el ambiente. Por otra parte, se deben considerar elementos fertilizantes que permitan optimizar el aprovechamiento por parte de la planta y reducir la lixiviación al suelo de residuos acumulables. El sílice (Si), no está considerado como un elemento esencial para las plantas, sin embargo, existen reportes de su eficacia para mejorar la producción de plantas ornamentales y su efecto en la respuesta a condiciones de estrés biótico y abiótico. Por ejemplo, en rosa miniatura, Datnoff, et al. (2006) y Larsen (2008), reportaron un incremento en la tolerancia a Mildiu; también se ha reportado reducción de incidencia en mancha negra (black spot) en rosa de corte (Gillman et al., 2003) y como aplicación foliar, McAvoy y Bible (1996) reportaron adecuado control para el ennegrecimiento del tallo. La acumulación de $\mathrm{Si}$ en las hojas de la nochebuena, en tratamientos con contenido mínimo de este elemento, fue reportado por Mattson y Leatherwood (2010), en adición, estos autores reportaron cambios en características morfológicas de este cultivo. El objetivo del presente trabajo, fue probar el efecto de tres tratamientos de fertilización, utilizando un producto a base de Silicato de potasio en parámetros de crecimiento y desarrollo de plantas de nochebuena de la variedad Freedom RED.

\section{Materiales y métodos}

\section{Material vegetal y diseño experimental}

Se utilizaron esquejes enraizados de nochebuena de la variedad Freedom $R E D$ producidos por una casa comercial del Estado de Morelos. El tamaño promedio de los esquejes fue de $11.5 \mathrm{~cm}$, con buen estado fitosanitario, tallos erguidos y sistema radicular vigoroso, coloración del follaje verde intenso con meristemo apical en desarrollo en el total de los individuos. Los esquejes fueron transplantados en un sustrato con la siguiente composición: $40 \%$ tierra de encino, $50 \%$ jal tamizada y $10 \%$ de composta de bagazo de caña.

Para probar el efecto de la adición de silicato de potasio, se utilizaron cuatro tratamientos según lo presentado en el Cuadro 1. Se utilizó una fertilización base aplicada en drench, según el protocolo propuesto por Argo y Biernbau (1995), 
consistente en la utilización de fertilizante ultrasoluble con concentraciones de Nitrógeno de $28 \mathrm{~mol} \mathrm{~m}^{-3}$ para las primeras tres semanas de producción, después de lo cual la concentración debe ser reducida a $21 \mathrm{~mol} \mathrm{~m}^{-3}$ hasta 2 semanas antes de la completa coloración de las brácteas.

Cuadro 1. Tratamientos utilizados en el presente estudio

\begin{tabular}{cl}
\hline Tratamiento & \multicolumn{1}{c}{ Descripción } \\
\hline Testigo & Fertilización drench \\
I & drench + foliar \\
II & drench + Sillisec K \\
III & drench + Sillisec K+ foliar
\end{tabular}

Se utilizó el producto Sillisec K a base de silicato de potasio y la fertilización foliar consistió en la aplicación de microelementos.

Las variables evaluadas fueron: Brotes después del pinch (BDP), Longitud de la raíz principal (LR), Área de hoja $(\mathrm{AH})$, Dosel (D) y número de inflorescencias (NF).

Las variables métricas fueron LR y AH. Las fechas de determinación correspondieron, el día 217, 249 y 281 del calendario juliano para 2019.

Análisis de datos

El modelado de respuesta de la nochebuena a tres tratamientos distintos, en comparación con un testigo estadístico, se realizó mediante el cálculo de la tasa de crecimientto de las raíces mediante la fórmula:

$$
\operatorname{tas} a=\frac{\ln x_{2}-\ln x_{1}}{t_{2}-t_{1}}
$$

Se calcularon los coeficientes de asimetría y curtosis en los datos para cada tratamientos para probar la normalidad de las variables. se realizaron pruebas de Kruskall-Wallis, una para cada fecha, para ambas variables. Finalmente se realizó una prueba de chi cuadrada para comparar los tratamientos con variables no métricas.

\section{Resultados y Discusión}

Análisis de las variables LR y AH

Las longitudes medias muestrales de las raíces al día 217 mostraron valores decrecientes del tratamiento III al testigo. La diferencia entre los promedios del tratamiento III y el promedio del testigo fue de $69.153 \mathrm{~cm}$. Sin embargo la varianza del tratamiento III también fue mucho mayor a la del testigo, en razón aproximada de 9.5:1.

Para la anchura de las hojas, el tratamiento I logró un promedio muestral mayor al de los otros tres, cuyos promedios son muy próximos. No hay evidencia de que las varianzas sean diferentes.

Para la segunda medición, hubo un aumento de la longitud de raíces para todos los tratamientos. Las tasas de crecimiento calculadas se presentan en el Cuadro 2.

La prueba de Kruskall-Wallis proporcionó evidencia de diferencias entre las longitudes de raíces de los tratamientos. Todos son estadísticamente diferentes. El que obtuvo la mejor respuesta es el tratamiento III, seguido del tratamiento II, posteriormente el tratamiento I, y finalmente el testigo. 
Cuadro 2. Tasa de crecimiento para las variables LR y AH en tratamientos de fertilización de nochebuena

\begin{tabular}{cccc}
\hline Variable/Tratamiento & Día juliano 217 & Día juliano 249 & $\begin{array}{c}\text { Tasa de crecimiento } \\
\text { del periodo }\end{array}$ \\
\hline $\begin{array}{c}\text { Longitud de raíces } \\
\text { Testigo }\end{array}$ & 30.020 & 37.374 & 0.0066604 \\
Tratamiento 1 & 46.855 & 73.91 & 0.0142435 \\
Tratamiento 2 & 63.17 & 89.949 & 0.0110442 \\
Tratamiento 3 & 93.92 & 125.39 & 0.0090308 \\
Anchura de hojas & & & \\
Testigo & 76.06 & 83.08 & 0.0027588 \\
Tratamiento 1 & 82.56 & 90.444 & 0.0028502 \\
Tratamiento 2 & 76.505 & 89.957 & 0.0050617 \\
Tratamiento 3 & 71.136 & 90.449 & 0.0075060 \\
\hline
\end{tabular}

La prueba de Kruskall Wallis proporciona evidencia de que no hubo diferencias significativas para la anchura de las hojas entre los tratamientos al día 281.

Análisis de las variables BDP, D y NF

A los 281 días, se manifestaron diferencias para la variable BDP entre los tratamientos. El que obtuvo la respuesta más alta y significativamente superior a los demás fue el tratamiento III, seguido por los tratamientos II y I, que resultaron iguales entre sí y superiores al testigo.

En las prueba tanto de KruskallWallis como chi cuadrada, para la variable $\mathrm{D}$ al día 281, se encontraron evidencias de diferencia estadística. El tratamiento III tuvo un dosel significativamente superior a todos los demás, seguido por el tratamiento II y I, que son iguales entre sí y superiores al testigo.

En el caso de la variable NF se encontró evidencia estadística de diferencias entre para el testigo y los tratamientos, siendo inferior para el testigo, en comparación con el de los tratamientos que produjeron cinco o más inflorescencias al día 281. Los resultados obtenidos, proporcionan información referente a los cambios morfológicos que se manifiestaron en las plantas sometidas a los diferentes tratamientos, lo cual ha sido reportado en trabajos como el realizado por Galicia-Jimenez et al. (2001) quienes proprobaron tratamientos con sombra.

\section{Conclusiones}

La precisión en la fertilización de nochebuena, es fundamental para la obtención de plantas cuya belleza impresione a los consumidores. El tamaño de la planta es una cuestion importante dentro de los parámetros de comercialización. En el presente trabajo se concluye que las aportaciones de fertilizante tanto en drench, como foliar adicionado con silicato de potasio, produjeron plantas con los mejores resultados. 


\section{Literatura citada}

Argo, R. G. y J.A. Biernbau (1995). The effect of irrigation method, watersoluble fertilization, replant nutrient charge, and surface evaporation on early vegetative and root growth of poinsettia. Journal of the American Society of Horticultural Science. 120(2): 163-169.

Datnoff, L.E., T.A. Nell, R.T. Leonard, y B.A. Rutherford (2006). Effect of silicon on powdery mildew development on miniature potted rose. Phytopathology. 96:S28.

Galicia-Jiménez, A.B., C. Trejo, L. A. Valdéz-Aguilar, M.T. RodríguezGonzález y C. B. Peña-Valdivia (2001). Shade intensity and its effect in morphology and physiology of poinsettia (Euphorbia pulcherrima Willd.). Revista Chapingo Serie Horticultura 7(2): 143-149.

Gillman, J.H., D.C. Zlesak, y J.A. Smith. (2003). Applications of potassium silicate decrease black spot infection in Rosa hybrida 'Meipelta' (Fuchsia Meidiland). HortScience 38:11441147.

Larsen, A.K. (2008). Less mildew in pot roses with silicon. FlowerTECH. 11:18-19.

Mattson, N.S. y W.R. Leatherwood (2010). Potassium silicate drenches increase leaf silicon content and afect morphological traits of several floriculture crops grown in a peatbased substrate. HortSicence. 45(1):43-47.

McAvoy, R.J. y B.B. Bible (1996). Silica sprays reduce the incidence and severity of bract necrosis in poinsettia. HortScience 31:1146-1149.

Schuch., U., R.A. Redak y J. Bethke (1995). Whole-plant responses of six poinsettia cultivars to three fertilizer and two irrigation regimes. Journal of the American Society of Horticultural Science. 121(1): 69-7. 